\title{
Heat transfer between shell and rigid body through the thin heat-conducting layer taking into account mechanical contact
}

\author{
V. V. Zozulya \\ Centro de Investigación Científica de Yucatán, Mérida, Yucatán, Mexico
}

\begin{abstract}
A problem of heat conducting and unilateral contact of a shell with a rigid body through the heat-conducting layer is formulated. An approach consists in considering a change of layer thickness in the process of the shell deformation. For modelling thermoelastic state of the shell classical Kirchhoff-Love's model is explored. For modelling heat conductivity of the shell expansion into a polynomial Legendre series in terms of the thickness is used. Contact conditions that take into account possibilities for unilateral mechanical contact and change of heat transfer conditions between shell and rigid body are formulated. Numerical examples of the unilateral thermoelastic contact of the cylindrical shells and rigid body through the heat-conducting layer are considered. Influence of physical and geometrical parameters of the shell and heat conducting layer is investigated.
\end{abstract}

Keywords: heat-conductivity, cylindrical shell, heat-conducting layer, mechanical contact.

\section{Introduction}

Contact interaction is the most common way to transfer load from one body to another. In the case of contacting bodies having different temperatures between them heat-contact interactions take place. Therefore, not only the condition of the mechanical contact, but also conditions of the thermal contact have to be considered. Usually, perfect thermal contact is assumed, i.e. it is assumed that the temperature and the thermal flux of the contacting bodies in the contact area are the same [3]. In numerous publications [2, 4-15] it was shown that in many cases these contact conditions are not acceptable because they can not take into 
account physical processes related to deformation and heat exchange. In these publications the problem of thermoelastic contact of plates and shells have been considered through a heat-conduction layer with considering change of the layer thickness during the plates and shells deformation. Numerical examples presented there show that in many important cases for science and engineering the result obtained using perfect thermoelastic contact conditions and the conditions with considering change of the layer thickness in the process of deformation are very different. In some cases the difference is not only quantitative but also qualitative. Therefore, it is very important to consider contact conditions which relate deformations and heat transfer in the problems where thin-walled structures may have contact though the heat-conducting layer in the intensive temperature field. Such kinds of problems take place in many important structures, equipment, and devices in chemical, airspace, nuclear industries, etc.

The developed approach have been applied to the plates and shells thermoelastic contact problems in [4-7, 11-15], the laminated composite materials with the possibility of delamination and thermoelastic contact in temperature field in $[8,9]$, and the pencil-thin nuclear fuel rods modeling in [10].

In this paper some new results related to unilateral thermoelastic contact of the axisymmetrical cylindrical shell through the heat-conducting layer are formulated. The connected equations of thermoelasticity and heat conductivity are created. These equations take into account change of the conditions of heat exchange between the shell and the rigid body during its deformation and possibility of close unilateral mechanical contact. Numerical examples of the heat conductivity of the cylindrical shells through the heat-conducting layer are considered. The thermomechanical effects caused by contact interaction and their influence on the thermomechanics parameters are investigated.

\section{Statement of the problem}

Let us consider an axisymmetrical cylindrical shell with parameters: $r$ is a radius, $2 h$ is a thickness, $l$ is a length, $\Omega, \Omega^{+}$and $\Omega^{-}$are middle, external and internal surfaces of the shell. We consider two situations illustrated in the fig.1: a) a rigid punch is placed inside of the shell with gap $h_{0}(x)$ b) a cylindrical shell placed inside of the cylindrical hole in the rigid body with gap $h_{0}(x)$.

The heat is transferred from the body surface $\psi$ to the shell and inversely through the heat conducting layer which is not resisted shell deformation. The shell can be subjected to external mechanical load $p(x)$ and temperature $T(x)$. Consequently, the shell is deformed and can come into mechanical contact with the rigid body. As a result is established as unknown before close contact area $\Omega_{e}=\Omega^{ \pm} \cap \psi$ and forces of contact interaction $g(x)$. Thus we have a situation with deformation influence on heat exchange and temperature influence on deformation.

In such formulation we have connected the thermoelastic and heat conducting problem. In this case, equations of thermoelasticity and heat conductivity cannot 
be solved separately. The possibility for unilateral mechanical contact have to be also taken into account.

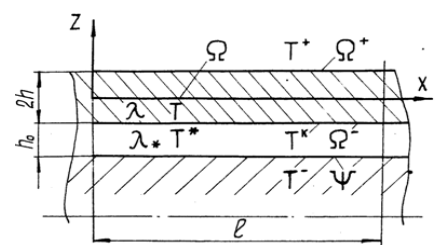

a)

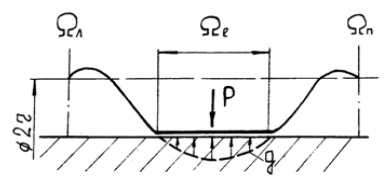

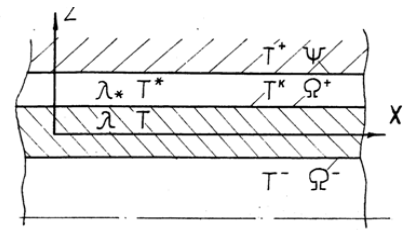

ס)

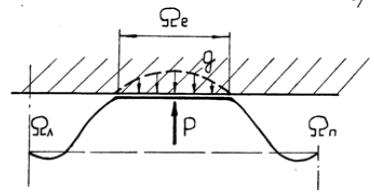

Figure 1.

\subsection{Equations of thermoelasticity of the shell}

We will use here a classical Kirchhoff-Love's shell theory. According to this theory axial $\varepsilon_{x}$ and circular $\varepsilon_{\theta}$ deformations of the shell middle surface are defined by the equations

$$
\varepsilon_{x}=\frac{d u+d v r}{d x}=\frac{d u}{d x}+\frac{d^{2} w}{d x^{2}} z, \varepsilon_{\theta}=\frac{2 \pi(r-z+w)-2 \pi(r-z)}{2 \pi(r-z)}=\frac{w}{r-z} \approx \frac{w}{r}
$$

and corresponding stresses by

$$
\begin{gathered}
\sigma_{x}=\frac{E}{1-v^{2}}\left[\frac{d u}{\partial x}+\frac{d^{2} w}{\partial x^{2}} z+v \frac{w}{r}-\alpha_{t}(1+v) T\right], \\
\sigma_{\theta}=\frac{E}{1-v^{2}}\left[v \frac{d u}{\partial x}+v \frac{d^{2} w}{\partial x^{2}} z+\frac{w}{r}-\alpha_{t}(1+v) T\right],
\end{gathered}
$$

where $u$ and $w$ are displacements in axial and circular directions, $E$ and $v$ are modulus of elasticity and Poison ratio, $T=T_{0}+T_{1} z$ is temperature of the shell. Differential equations of thermo-elasticity have the form

$$
\frac{d^{4} w}{d x^{4}}+4 \beta^{4} w=\frac{1}{D}(p-g)-a_{0} T_{0}+a_{1} \frac{d^{2} T_{1}}{d x^{2}},
$$

where $\beta^{4}=\frac{3\left(1-v^{2}\right)}{4 h^{2} r^{2}} a_{0}=\frac{3 \alpha_{t}\left(1-v^{2}\right)}{h^{2} r}, a_{1}=\frac{\alpha_{t}(1+v)}{h}$

\subsection{Equations of heat conductivity of the shell}

Following [4] we represent temperature of the shell in the form

$$
T=T_{0}+\frac{z}{h} T_{1}, T_{0}=\frac{1}{2 h} \int_{-h}^{h} T d z, T_{1}=\frac{3}{2 h^{2}} \int_{-h}^{h} T z d z
$$


Then differential equations of heat conductivity have the form

$$
\begin{gathered}
\frac{d^{2} T_{0}}{d x^{2}}+\frac{1}{2 h}\left(Q^{+}-Q^{-}\right)-\frac{1}{r} Q_{0}=0 \\
\frac{d^{2} T_{1}}{d x^{2}}+\frac{3}{2 h}\left(Q^{+}+Q^{-}\right)-\frac{3}{h} Q_{0}+\frac{1}{r} Q_{1}=0
\end{gathered}
$$

Here $T_{i}$ and $Q_{i}$ are coefficients of the polynomial expansion of the temperature and heat flux. They are related by equations

$$
\begin{gathered}
Q_{0}=\frac{1}{2 h}\left(T^{+}-T^{-}\right), Q_{1}=\frac{3}{2 h}\left(T^{+}+T^{-}\right), Q_{2}=5 Q_{0}-\frac{5}{h} T_{1}, \\
Q^{+}+Q^{-}=2\left(Q_{0}+Q_{2}\right), Q^{+}-Q^{-}=2 Q_{1}
\end{gathered}
$$

where $T^{+}, T^{-}, Q^{+}$and $Q^{-}$are their values on the surfaces $\Omega^{+}$and $\Omega^{-}$ respectively.

\subsection{Boundary and contact conditions}

For finite length shell we consider the following mechanical boundary conditions.

$$
\begin{aligned}
\left.\frac{d^{3} w}{d x^{3}}\right|_{\Omega^{L}, \Omega^{p}}=0,\left.\frac{d^{2} w}{d x^{2}}\right|_{\Omega^{L}, \Omega^{p}} & =0 \text { - free end; }\left.w\right|_{\Omega^{L}, \Omega^{p}}=0,\left.\frac{d w}{d x}\right|_{\Omega^{L}, \Omega^{p}}=0 \text { - fixed end; } \\
\left.w\right|_{\Omega^{L}, \Omega^{p}} & =0,\left.\frac{d^{2} w}{d x^{2}}\right|_{\Omega^{L}, \Omega^{p}}=0 \text { - simply supported end. }
\end{aligned}
$$

For infinite length shell we consider the following conditions at infinity

$$
w \rightarrow 0, \frac{d w}{d x} \rightarrow 0, \frac{d^{2} w}{d x^{2}} \rightarrow 0, \frac{d^{3} w}{d x^{3}} \rightarrow 0 \text { for } x \rightarrow \infty
$$

Boundary conditions for the equations of heat conductivity are

$$
\begin{gathered}
\left.T\right|_{\Omega^{L}}=T^{L}-\text { if temperature is prescribed; } \\
\left.\lambda \frac{d T}{d x}\right|_{\Omega^{L}}=q^{L} \text { - if heat flux is prescribed; } \\
\frac{\partial T}{\partial x}+\left.\frac{\alpha}{\lambda}\left(T-T^{L}\right)\right|_{\Omega^{L}}=0 \text { - for convective heat transfer }
\end{gathered}
$$

where $\lambda$ is a coefficient of heat conductivity, $\alpha$ is a coefficient heat exchange though a surface.

Mechanical and thermal contact conditions are presented in the form [4-7]

$$
\begin{gathered}
w<h_{0} \rightarrow g=0, \forall x \in \Omega^{+} \backslash \Omega_{e} \quad, w=h_{0} \rightarrow g>0, \forall x \in \Omega_{e} \\
T=T_{k}, T_{k}=\frac{\lambda\left(h_{0}-w\right) T^{+}+\lambda_{*} h T^{-}}{\lambda\left(h_{0}-w\right)+\lambda_{*} h}
\end{gathered}
$$

It is important to mention that thermal contact conditions include shell deflection $w$ nonlinearly and therefore equation (10) relates the equations of shell thermoelasticity (3) and heat conductivity (5). As a result we have 
connected nonlinear problems of thermoelasticity and heat conductivity with unilateral contact conditions.

\subsection{Transformation to the integral equation}

In $[4,6,7,13-15]$ it was shown that the differential equations of the shell thermoelasticity (3) and heat conductivity (5) can be transformed into the integral equations of Hammerstein's type

$$
\begin{aligned}
& \int_{l} G_{0}(x, y) F_{0}(y) d y=T^{0}, \int_{l} G_{1}(x, y) F_{1}(y) d y=T^{1} \\
& \int_{l} W(x, y)\left\{\frac{1}{D}[p(y)-g(y)]-\beta_{0} F_{3}(y)\right\} d y=w,
\end{aligned}
$$

where

$$
\begin{gathered}
F_{0}=0.5 \varepsilon_{0}\left(T^{-}+T_{k}\right)+\frac{1}{2 h r}\left(T_{k}-T^{-}\right), F_{1}=0.5 \varepsilon_{1}\left(T_{k}-T^{-}\right)+\frac{3}{2 h r}\left(T_{k}+T^{-}\right)-\frac{3}{h r} T^{1}, \\
F_{3}=\beta_{1}\left(F_{1}+\varepsilon_{1}^{2} T^{1}\right)-\beta_{0} T^{0}
\end{gathered}
$$

The kernels in these integral equations are fundamental solutions for corresponding differential operators. They have the form

$$
\begin{array}{r}
G_{0}(x, y)=\exp \left(-\varepsilon_{0}|x-y|\right) / 2 \varepsilon_{0}, G_{1}(x, y)=\exp \left(-\varepsilon_{1}|x-y|\right) / 2 \varepsilon_{1} \\
W(x, y)=\frac{1}{8 \beta^{3} D} \exp (-\beta|x-y|)[\cos (\beta|x-y|)+\sin (\beta|x-y|)]
\end{array}
$$

where

$$
\varepsilon_{0}=\frac{3}{h^{2}}, \varepsilon_{1}=\frac{15}{h^{2}}, \beta_{0}=\frac{3(1-v) \alpha_{\tau}}{h^{2} r}, \beta_{1}=\frac{(1+v) \alpha_{\tau}}{h}, D=\frac{2 E h^{3}}{3\left(1-v^{2}\right)}
$$

\subsection{Algorithm for the problem solution}

Algorithms for the problem's solution consists in an iterative process of the nonlinear integral equations of Hammerstein's type solution and in the case if unilateral contact taking place an additional iterative algorithm is used. The algorithm has been elaborated in $[4,6]$. In the problems under consideration the algorithm is convergent and convergence is fast enough.

\subsection{Stress calculation}

Stresses in the axisymmetrical cylindrical shell are calculated by formulas (2), which for convenience may be presented in the form

$$
\begin{gathered}
\sigma_{x}=\frac{E}{1-v^{2}}\left[\frac{d^{2} w}{d x^{2}} z-(1+v) \alpha_{t} T^{1} \frac{z}{2 h}\right], \\
\sigma_{\theta}=\frac{E w}{r}-\alpha_{t} T^{0} E+\frac{E}{1-v^{2}}\left[v \frac{d^{2} w}{d x^{2}} z-(1+v) \alpha_{t} T^{1} \frac{z}{2 h}\right]
\end{gathered}
$$


Deriving from (12) expression for the second derivative of the displacements and substituting it into (14) we obtain the following integral representations for stress

$$
\begin{gathered}
\sigma_{x}(x)=b_{0} \int_{0}^{l} T^{0}(\xi) \frac{d^{2} G(\xi, x)}{d x^{2}} d \xi+ \\
b_{1} \int_{0}^{l} G(\xi, x) T^{1}(\xi) d \xi-b_{2} T^{1}(x)+b_{3} \int_{0}^{l}[p(\xi)-q(\xi)] \frac{d^{2} G(\xi, x)}{d x^{2}} d \xi \\
\sigma_{\theta}(x)=v \sigma_{x}(x)+\frac{E w(x)}{r}-\alpha_{t} E\left[T^{0}(x)+T^{1}(x) z\right]
\end{gathered}
$$

where

$$
\begin{aligned}
& b_{0}=\frac{3 E \alpha_{t} z r^{5}}{(1+v) h^{2} h_{0}}, b_{1}=\frac{3(1+v) E \alpha_{\tau} r^{6} z}{4 h^{3} h^{2}{ }_{0}}, b_{2}=\frac{E \alpha_{\tau} r^{4} z}{(1-v) h h_{0}}, \\
& b_{3}=\frac{3 r^{6} z}{2 h^{3} h_{0}}, \beta_{0}=\frac{3 \alpha_{t} E r z}{h^{2}}, \beta_{1}=\frac{3(1-v) \alpha_{t} E r^{2} z}{h^{2}}, \beta_{3}=\frac{r^{2} z}{8 h^{3}} .
\end{aligned}
$$

Mizes stresses are calculated by the equations

$$
\begin{gathered}
\sigma_{i}^{-}=\sqrt{\left(\sigma_{x}^{-}\right)^{2}+\left(\sigma_{x}^{-}\right)\left(\sigma_{0}^{-}\right)+\left(\sigma_{0}^{-}\right)^{2}} \\
\sigma_{i}^{+}=1 / \sqrt{2} \sqrt{\left(\sigma_{x}^{+}-q\right)^{2}+\left(\sigma_{x}^{+}-\sigma_{x}^{+}\right)^{2}+\left(\sigma_{0}^{+}+q\right)^{2}}
\end{gathered}
$$

Using equations (15) and (16) stresses in the shell can be easy calculated.

\section{Investigation of the thermo-mechanical state of the shell}

We will consider here thermo-mechanical state of the shell with considering influence of the shell deformation on the heat transfer between it and a rigid body. Calculation have been done for the data: material properties: $E=2.5 \cdot 10^{5} \mathrm{MPa}, \quad v=0.25 \quad \alpha_{\tau}=2.5 \cdot 10^{-5} 1 /{ }^{o} \mathrm{C} \quad, \quad \lambda_{*}=1 \mathrm{~V} / \mathrm{m}^{o} \mathrm{C}$ and geometrical parameters $r=0.5 \mathrm{~m}, h=0.01 \mathrm{~m}, l_{b}=r$,

Example 1. We consider an axisymmentrical cylindrical shell of infinite length placed into the rigid stirrup with a homogeneous initial gap as is shown in fig.2. Temperature on the stirrup surface is not homogeneous and equal to

$$
T^{+}(x)=T_{m}+T_{b} \sin \pi x / l_{b}, T_{b}=600^{\circ} C T_{m}=100^{\circ} \mathrm{C} .
$$

On the shell surface act homogeneous load $p(x)=10 M P a$, temperature on the internal surface of shell is $T^{-}=0^{\circ} C$, initial gap is equal to $h_{0}=0.5 h$ and $\lambda_{1}=2 \lambda_{*}$.

In fig. 3 are presented: Mizes stresses on external $\sigma^{+}$and internal $\sigma^{-}$ surfaces of the shell, force of contact interaction $g$, normalized bending $W=w / h_{0}$ and temperature on contact surface $T_{k}$. The dashed lines correspond to a solution for perfect thermal contact without counting influence of the shell deformation on the heat exchange and the solid lines correspond to the solution presented here. 


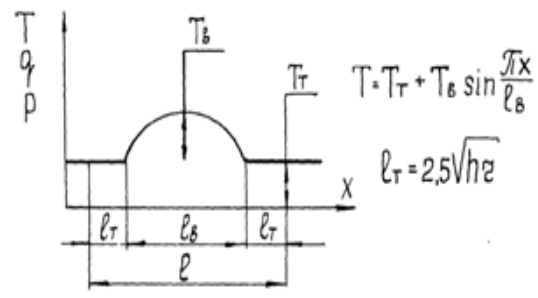

Figure 2.

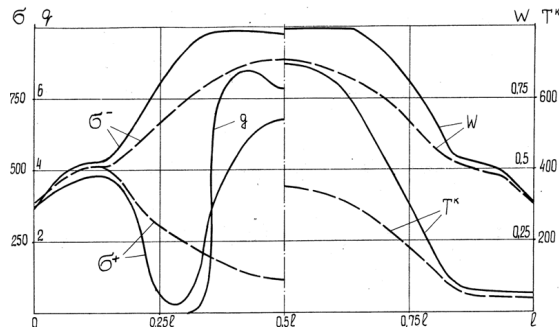

Figure 3.

Mizes stresses on external $\sigma^{+}$and internal $\sigma^{-}$surfaces of the shell, force of contact interaction $g$, for the same data and initial gap $h_{0}=0.1 h$ are presented in fig 4 and 5 external and internal punch respectively.

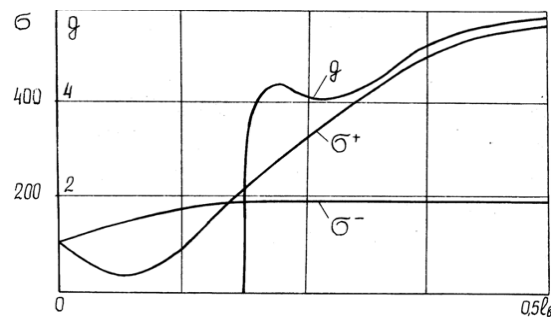

Figure 4.

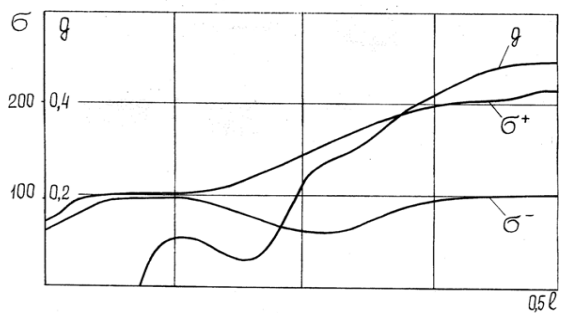

Figure 5 .

Analysis of these data shows that no homogeneous temperature distribution cause significant shell deformations and close mechanical contact with a rigid stirrup. As a result significant stress occurs in the shell. Calculations assuming perfect thermal contact lead to significant inaccuracy, which is not only quantitative but also qualitative.

Example 2. Here we consider an axisymmentrical cylindrical shell of infinite length placed into the rigid stirrup with no homogeneous initial gap, as shown in the Fig.6.

The gap is given by the function

$$
h_{*}(x)=h_{o}+h_{b} \sin \pi x / l_{b}, h_{0}=0.5 h, h_{b}=h_{0} / 2, l_{b}=r .
$$

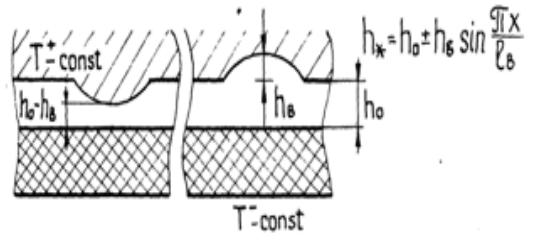

Figure 6.

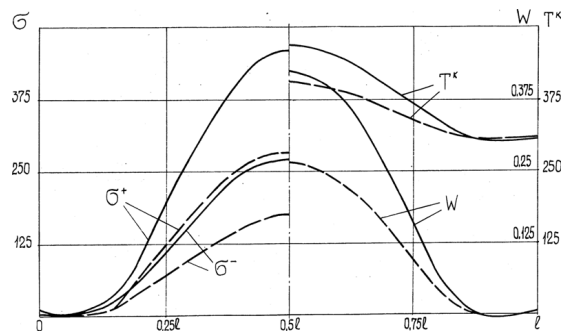

Figure 7. 
In fig.7 are presented: Mizes stresses on external $\sigma^{+}$and internal $\sigma^{-}$ surfaces of the shell, normalized bending $W=w / h_{0}$ and temperature on contact surface $T_{k}$. The dashed lines correspond to solution for perfect thermal contact without counting influence of the shell deformation on the heat exchange and the solid lines correspond to the solution presented here. Analysis of these data shows, that no homogeneous initial gap cause significant shell deformations. As a result, in the shell, no significant homogeneous stress and temperature distribution occurs. Calculations assuming perfect thermal contact lead to significant inaccuracy. Some values of thermomechanical state differ.

Example 3. We also investigate influence of different parameters on shell deformation and heat exchange. In fig. 8 are presented deformations and temperature on the contact surface for fixed-end shell in homogeneous temperature field $T^{-}=150^{\circ} C, T^{+}=0^{\circ} C$, for $\lambda_{1}=4 \lambda_{*}, h_{0}=0.25 h$.

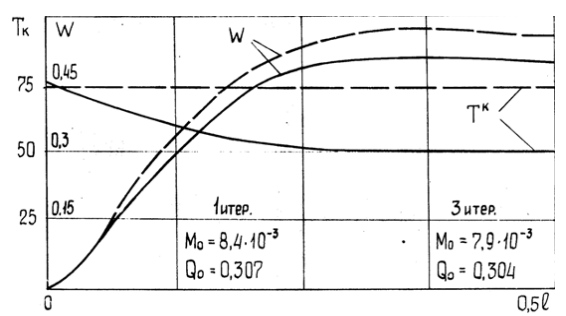

Figure 8 .

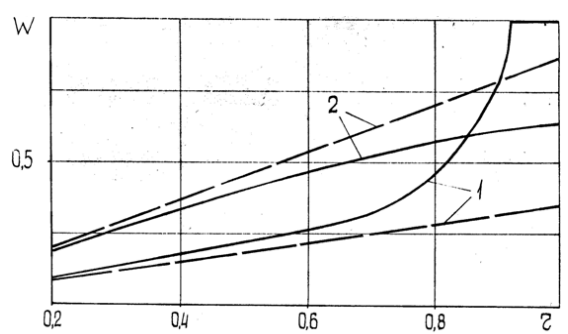

Figure 9.

It is interesting to point out that in the case when $T^{-}>T^{+}$accounting influence of the shell deformation on the heat exchange decreases its deformation, stress and temperature distributions.

In fig. 9 is presented dependence of the shell deflection on its radius. Calculations have been done for the following data: $\lambda_{1}=5 \lambda_{*} h_{0}=0.5 h$, curve 1 for the external punch and $T_{b}=600^{\circ} \mathrm{C}$, curve 2 for the internal punch and $T_{b}=200^{\circ} \mathrm{C}$. It is important to mention that for external punch when $r / h>50$ dependence becomes nonlinear and the shell is attracted to the punch, solid curve 1 .

All the above calculations have been done for the case when on the surfaces $\Omega$ and $\psi$ is prescribed temperature. In fig. 10 and fig. 11 dependences $w=f\left(\lambda_{1} / \lambda_{*}\right)$ and $w=f\left(h_{0} / h\right)$ for the external punch are presented. Curves 1 correspond to the case when on the surfaces $\psi$ temperature and on the surface $\Omega$ heat flux are set. Curves 2 correspond to the case when on the surfaces $\Omega$ temperature and $\psi$ on the surface heat flux are set. In both cases temperature is distributed in accordance with equation (17), heat transfer rate is equal to $q=-2 \cdot 10^{4}\left[\mathrm{~V} / \mathrm{m}^{2} \mathrm{~K}\right]$ and $q=1.4 \cdot 10^{4}\left[\mathrm{~V} / \mathrm{m}^{2} \mathrm{~K}\right]$ for the first and second cases 
respectively. In the first case shell deflection is minimal for $\lambda_{1} / \lambda_{*}=1$ and increases when $h_{0} / h$ decrease. In the second case shell deflection is maximal for $\lambda_{1} / \lambda_{*}=1$ and does not depend much on $h_{0} / h$. In both cases shell deflection does not change much for $\lambda_{1} / \lambda_{*}>10$.

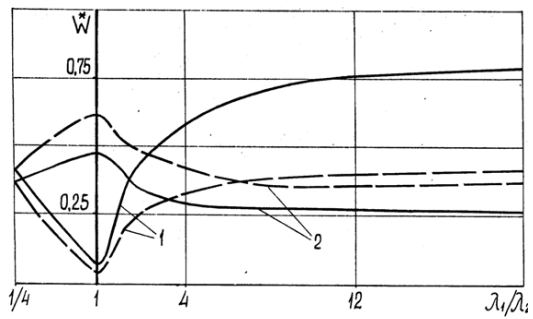

Figure 10.

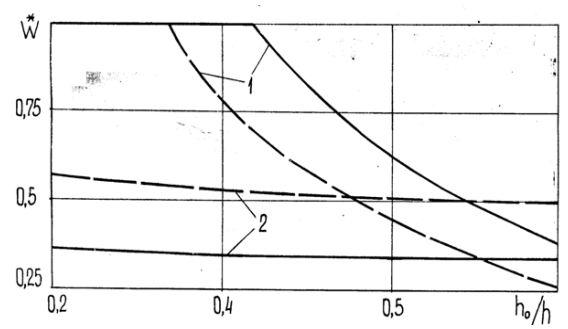

Figure 11.

\section{Conclusions}

The results presented here show that in many situations thin-walled structures subjected to high temperature perfect thermal contact conditions are not acceptable because they cannot take into account physical processes related to deformation and heat exchange. Such kinds of problems may occur in different field of science and engineering, for example in nuclear, aerospace, chemical industries, etc. In such situations the approach developed here and in our previous publications have to be used.

\section{References}

[1] Boley B.A. and Weiner J.H. Theory of thermal stress, Wiley, New York, 1960.

[2] Kantor B. Ya., Zozulya V.V. Connected problem on contact plate with rigid body though the heat-conducting layer, Doclady Akademii Nauk Ukr. SSR, 4, pp. 31-33, 1988. (in Russian)

[3] Postrigach Ya. S, Shvets P. N. Yu. M., Thermoelasticity of thin shells, Kiev, Naukova dumka, 1978. (in Russian)

[4] Zozulya V.V. Contact cylindrical shell with a rigid body though the heatconducting layer, Doclady Akademii Nauk Ukr. SSR, 1989, 10, pp.48-51. (in Russian)

[5] Zozulya V.V. The combined problem of thermo- elastic contact between two plates though a heat conducting layer, Journal Applied Mathematics and Mechanics, 53(5), pp.722-727, 1989.

[6] Zozulya V.V. Bending of a plate in temperature field under restrictions, Izvestiya vuzov. Engineering, 1, pp. 24-27, 1990. (in Russian) 
[7] Zozulya V.V. Contact cylindrical shell with a rigid body though the heatconducting layer in transitional temperature field, Mechanics of Solids, Vol. 2, pp.160-165, 1991.

[8] Zozulya V. V. Nonperfect contact of laminated shells with considering debonding between laminas in temperature field, Theoretical and Applied Mechanics, Vol. 42, pp.92-97, 2006.

[9] Zozulya V.V. Laminated shells with debonding between laminas in temperature field, International Applied Mechanics, Vol. 42(7), pp. 135 $141,2006$.

[10] Zozulya V.V. Mathematical Modelling of Pencil-Thin Nuclear Fuel Rods, in Proc. SMIRT19 Conf. Structural Mechanics in Reactor Technology, A. Gupta Ed., Toronto, 2007, pp. C04-C12.

[11] Zozulya V.V. Contact of a shell and rigid body though the heat-conducting layer temperature field, International Journal of Mathematical Models and Methods in Applied Sciences, 2(1), pp.138-145, 2007.

[12] Zozulya V.V. Contact of the thin-walled structures and rigid body though the heat-conducting layer, in Proc. Theoretical and Experimental aspects of heat and mass transfer, Acapulco, Mexico, pp. 145-150, 2008.

[13] Zozulya V.V., Aguilar M., Thermo-elastic contact and heat transfer between plates through the heat-conducting layer, in Heat transfer 2000, B. Sunden and C.A. Brebbia Eds. Computational Mechanics Publications, Southampton, UK and Boston, USA, pp. 123-132, 2000.

[14] Zozulya V.V., Borodenko Yu. N. Thermoelastic condition of cylindrical shell, which interaction with a rigid body though the heat-conducting layer, Izvestiay vuzov. Engineering, 8, pp. 47-52, 1990. (in Russian)

[15] Zozulya V.V., Borodenko Yu. N. Connecting problem on contact of cylindrical shells with a rigid body in temperature though the heatconducting layer, Doclady Akademii Nauk Ukr. SSR, 4, pp.35-41, 1992. (in Russian). 